\title{
Physico-Chemical Characteristics of Nalban Wetland of East Kolkata Wetlands, A Ramsar Site, West Bengal, India
}

\author{
Ranga Ram Mohan ${ }^{1}$, T.S. Nagesh ${ }^{2}$, Anish Das², Sandeep Sahu², \\ D. Ravindra Kumar Reddy ${ }^{1}$ and Anand Prasad Paturi ${ }^{1}$
}

\author{
${ }^{1}$ College of Fishery Science, Muthukur, Nellore dt., Andhra Pradesh, India \\ ${ }^{2}$ Faculty of Fishery Sciences, Chakgaria, WBUAFS, Kolkata, West Bengal, India
}

*Corresponding author

\section{A B S T R A C T}

\section{Keywords \\ East Kolkata, Nalban wetland, Water quality, Soil quality, Tolerable limits, Ecological integrity, Threats \\ Article Info \\ Accepted: \\ 10 March 2019 \\ Available Online: \\ 10 April 2019}

The important physico-chemical parameters of water such as temperature, $\mathrm{pH}$, dissolved oxygen, free carbon dioxide, total alkalinity, total dissolved solids, electrical conductivity, Biological oxygen demand, nitrate-nitrogen, ammonia-nitrogen, phosphate-phosphorous and soil quality parameters such as $\mathrm{pH}$ and organic carbon were studied in Nalban wetland in triplicate by standard methods. Two-way analysis of variance (ANOVA) was performed to test the significant differences, if any, in the mean values of physcio-chemical parameters of water and sediment among months and sampling sites after confirmation of normality and homogeneity of variance (Zar, 1999). Tukey (HSD) test (Tukey, 1977) was used to evaluate pair-wise multiple comparisons. The difference was considered statistically significant at $P \leq 0.05$. All statistical analyses were performed using IBM SPSS 20.0 statistical software. All physico-chemical parameters were found to be within the tolerable limits of fishes that inhabit the wetland.

\section{Introduction}

Wetlands have been identified as one of the key life supporting ecosystems on this planet. They are considered as the most productive ecosystems as they constitute huge floral as well as faunal diversities (Chase, 2007). They also play a very important role in socioeconomic condition of the concerned region as they are used for aquaculture activities at commercial level (Williams, 1990), crucial for biodiversity conservation (Panthi et al.,
2014) and maintain the ecological integrity. Kolkata is sustained by this unique and friendly water regime which is in totality named as East Kolkata Wetlands (EKW) (Latitude $22^{\circ} 33^{\prime}-22^{\circ} 40^{\prime} \mathrm{N}$; Longitude $88^{\circ} 25^{\prime}$ $\left.88^{\circ} 35^{\prime} \mathrm{E}\right)$. East Kolkata Wetlands was declared as Ramsar site on $19^{\text {th }}$ August 2002 by Ramsar Convention Bureau. Wetlands provide an environment where photosynthesis can occur and the recycling of nutrients can take place apart from playing a significant role in supporting food chains (Adams, 1988). 
Within a wetland, the environmental characteristics are determined mostly by hydrological processes which display diurnal, seasonal and even annual fluctuations in response to meteorological, socioeconomic and geographical factors (Mould et al., 2010). Therefore the present study was carried out to determine physico-chemical characteristics of selected wetland.

\section{Materials and Methods}

\section{Site selection}

The sampling station called Nalban wetland (Latitude 22 $\quad 24^{\circ} 3.36 " \mathrm{~N}$; Longitude $88^{\circ} 25^{\prime} 41.02^{\prime \prime E}$ ) has been selected to analyse physico-chemical parameters

\section{Study Period}

The study was carried out for a period of six months (September 2016 to February 2017) for analysis of physico-chemical parameters of water and sediment.

\section{Collection of data}

Water and sediment samples were collected on site the Nalban wetland in three different sites viz., site 1(outlet), site 2 (middle) and site 3 (inlet) on monthly basis between morning 7.30 A.M. and 9.00 A.M. on sampling day.

\section{Analysis of physico-chemical parameters of water}

The physico-chemical parameters of water such as temperature, transparency, $\mathrm{pH}$, dissolved oxygen, free carbon dioxide, alkalinity, hardness, total dissolved solids, electrical conductivity, biological oxygen demand, nitrate-nitrogen, ammonia and phosphorus were estimated in triplicate by standard methods as described below.

\section{Statistical analysis}

Two-way analysis of variance (ANOVA) was performed to test the significant differences, if any, in the mean values of physico-chemical parameters of water and sediment among months and sampling sites after confirmation of normality and homogeneity of variance (Zar, 1999). Tukey (HSD) test (Tukey, 1977) was used to evaluate pair-wise multiple comparisons.

The difference was considered statistically significant at $P \leq 0.05$. All statistical analyses were performed using IBM SPSS 20.0 statistical software.

\section{Results and Discussion}

\section{Water temperature $\left(0^{\circ} \mathrm{C}\right)$}

In the present study, an overall minimum and maximum water temperature were observed between $21.10 \pm 0.10$ (site 1 and 2) in the month of December and 31.10 \pm 0.10 (site 2) in the month of September, respectively (Table 2).

This range of water temperature was found satisfactory and also ascertained by several workers (Ayyappan, 2006, Dampin et al., 2012; Mandal et al,. 2010; Jena and Das, 2011;) in sewage fed fish ponds in East Kolkata wetlands and different areas in India.

\section{Transparency $(\mathrm{cm})$}

In the present investigation it was revealed that the minimum and maximum transparency values varied from $19.10 \pm 0.17$ in the month of February to $28.47 \pm 0.15 \mathrm{~cm}$ in the month of September (Table 3) and these results are within ranged and strongly evident from work done by Basu et al., (2013) on same water body transparency, who reported transparency value ranging between $8.0-54.2 \mathrm{~cm}$. 


\section{Water $\mathbf{p}^{\mathbf{H}}$}

The range of water $\mathrm{pH}$ observed in present study generally remained within the range observed by Nwabueze (2013); Mandal et al., (2010); Dasgupta et al., (2008); Santra and Deb (1996) in different sewage fed ponds in West Bengal. The present $\mathrm{pH}$ results corroborated results (7.1 to 8.0) (Table 4) reported by Sondhia (2008) in Nalban wetland.

\section{Dissolved oxygen (mg/l)}

The dissolved oxygen levels of Nalban wetland showed minimum (7.28 \pm 0.04$)$ in the month of November and maximum (8.52 \pm 0.10$)$ during the month of January (Table 5). The present results are within the tolerable limits of fishes and the range recorded by different workers in East Kolkata Wetlands. Dasgupta et al., (2008) recorded dissolved oxygen varying from 5.2 to 10.2 $\mathrm{mg} / \mathrm{l}$ in sewage fed fish pond. The dissolved oxygen level of wastewater fish pond was 9.6 \pm 3.4 as recorded by Dampin et al., (2012) in Thailand. The fluctuation of dissolved oxygen within the level of 5.6 to $12.4 \mathrm{mg} / \mathrm{l}$ in sewage fed fish pond in Kolkata had been reported by Santra and Deb (1996).

\section{Free carbon dioxide (mg/l)}

The present results indicated that free carbon dioxide fluctuated from $8.20 \pm 0.10$ to $16.30 \pm 0.10 \mathrm{mg} / \mathrm{l}$ in site 1 and $8.27 \pm 0.12$ to $17.20 \pm 0.10 \mathrm{mg} / \mathrm{l}$ in site 2 and $8.83 \pm 0.15$ to $16.43 \pm 0.15 \mathrm{mg} / \mathrm{l}$ in site 3 (Table 6).

Bhatnagar et al., (2004) suggested $5-8 \mathrm{mg} / \mathrm{l}$ is essential for photosynthetic activity; 12-15 $\mathrm{mg} / \mathrm{l}$ is sub lethal to fish and $50-60 \mathrm{mg} / \mathrm{l}$ is lethal to fish. However, Boyd and Lichtkoppler (1979) was of the opinion that fish can survive up to $60 \mathrm{mg} / \mathrm{l}$ free carbon dioxide concentration.

\section{Total alkalinity (mg/l)}

Alkalinity levels recorded in the present study, thus, seem to be conducive for fish growth. Mandal et al., (2010) observed the range of total alkalinity from 122.8 to 167.2 $\mathrm{mg} / \mathrm{l}$ in water sample of sewage fed fish pond. Dampin et al., (2012) recorded the total alkalinity of $121 \pm 19.16 \mathrm{mg} / \mathrm{l}$ in sewage fed tilapia fish pond in Thailand. The total alkalinity from 149 to $240 \mathrm{mg} / \mathrm{l}$ was observed in Kolkata sewage fed fish pond by Mukherjee (2011) which is in the similar range recorded in the present study (Table 7).

\section{Total hardness (mg/l)}

During the present investigation total hardness levels were found to be in the range of $249.33 \pm 4.04 \mathrm{mg} / \mathrm{l}$ in the month of September to $296.33 \pm 4.04 \mathrm{mg} / \mathrm{l}$ in the month of February (Table 8).

Thus, it appears that hardness is slightly higher than the optimum levels. In treated waste water, hardness varied from $560 \mathrm{mg} / \mathrm{l}$ to 770 mg/l (Chattopadhyay, 2002). Similar results have been observed by Mukharjee (2011) and Dampin et al., (2012) in the range of $171 \pm 38.77 \mathrm{mg} / \mathrm{l}$ and 166 to $256 \mathrm{mg} / \mathrm{l}$ respectively in sewage fed fish ponds in Kolkata.

\section{Total dissolved solids (mg/l)}

The total dissolved solids of Nalban sewage fed pond ranged from $341.00 \pm 6.00 \mathrm{mg} / \mathrm{l}$ to $463.67 \pm 6.66 \mathrm{mg} / \mathrm{l}$ during the study period (Table 9). Dampin et al., (2012) recorded the total suspended solid value of $68.65 \pm 21.39$ $\mathrm{mg} / \mathrm{l}$ in sewage fed tilapia fish pond in Thailand. Mukharjee (2011) reported total suspended solids and total dissolved solids in the range between 52 to $107 \mathrm{mg} / \mathrm{l}$ and 327 to $589 \mathrm{mg} / \mathrm{l}$, respectively, which is corroborated with the present study. 


\section{Electrical conductivity $(\boldsymbol{\mu s} / \mathrm{Cm})$}

The electrical conductivity of Nalban sewage fed water body was ranging between $1076.00 \pm 9.85 \mu \mathrm{s} / \mathrm{Cm}$ (minimum in the month of November) and $1267.33 \pm 4.04 \mu \mathrm{s} / \mathrm{Cm}$ (maximum in the month of December) during study period (Table 10). This might be due to heavy discharge of sewage in the month of December. These results are in comparable with results were observed by various workers (Datta and Bhagwati, 2007; Hulyal and Kaliwal, 2011; Ramulu and Benarjee, 2013).

\section{Biological Oxygen Demand (BOD)}

During the present investigation the BOD level was found to be minimum $(16.87 \pm 1.67$ $\mathrm{mg} / \mathrm{l})$ in the month of September, while the maximum concentration $(33.17 \pm 1.04 \mathrm{mg} / \mathrm{l})$ was seen in the month of January (Table 11). Thang and Yen (2003) recorded similar pattern of variation in sewage fed fish ponds in Vietnam. Due to the highly discharging of organic loaded sewage and high microbial activity BOD level in the water body was elevated. Tanner et al., (1995) also stated that the higher values of BOD during rainy season was due to input of organic wastes and enhanced bacterial activity.

\section{Nitrate-Nitrogen (mg/l)}

The nitrate-nitrogen recorded in Nalban sewage wetland was very low and ranged between $0.053 \pm 0.008 \mathrm{mg} / \mathrm{l}$ (minimum) to $0.145 \pm 0.041 \mathrm{mg} / \mathrm{l}$ (maximum) (Table 12). The minimum value observed in the month of January and the maximum value observed during December month. Such low levels of nitrate-nitrogen ranging between 0.05 and 2.2 $\mathrm{mg} / \mathrm{l}$ was also recorded in the sewage fed aquaculture system by Ghosh et al., (1974).

\section{Ammonia-Nitrogen}

The ammonium-nitrogen was generally ranging from $0.29 \pm 0.02 \mathrm{mg} / \mathrm{l}$ to $0.52 \pm 0.06$ $\mathrm{mg} / \mathrm{l}$ (Table 13) during the study period. In site wise variation, site 3 showed significant $(\mathrm{p}<0.05)$ difference with the site 1 and site 2 . In monthly variation September and February months showed significant $(\mathrm{p}<0.05)$ difference in between them and also with rest of months.

Table.1 Standard methods

\begin{tabular}{|c|c|}
\hline Parameter & Instrument/method followed \\
\hline Temperature $\left(0^{\circ} \mathrm{C}\right)$ & Centigrade thermometer (Range: $0^{\circ} \mathrm{C}$ to $50^{\circ} \mathrm{C}$ ) \\
\hline Transparency (cm) & Secchi disc (20 cm diameter) \\
\hline pH & Digital pH meter (Hanna, Portugal) \\
\hline Dissolved oxygen (DO) (mg/lit) & Azide modification method (APHA, 1998) \\
\hline Free carbon dioxide (mg/lit) & Titrating method (APHA, 1995) \\
\hline Total alkalinity (mg/lit) & Titration method (APHA, 1998 \\
\hline Total hardness (mg/lit) & Titrating method (APHA, 1995) \\
\hline Total dissolved solids (TDS) & TDS meter (HiMedia) \\
\hline Electrical conductivity $(\boldsymbol{\mu s} / \mathbf{C m})$ & Digital conductivity meter (Make: Systronics, Model: 306) \\
\hline Biological oxygen demand (BOD) (mg/lit) & APHA (1998) method \\
\hline Nitrate-nitrogen $\left(\mathrm{NO}_{3}-\mathrm{N}\right)(\mathrm{mg} / \mathrm{lit})$ & EPA approved Brucine Method (Bain, 2009) \\
\hline Ammonia-nitrogen $\left(\mathrm{NH}_{3}-\mathrm{N}\right)(\mathrm{mg} / \mathrm{lit})$ & Phenate method (APHA, 1998). \\
\hline Phosphate-phosphorous $\left(\mathrm{PO}_{4}-\mathrm{P}\right)(\mathrm{mg} / \mathrm{lit})$ & Stannous chloride method (APHA, 1998). \\
\hline Soil pH & pH meter (Make: Systronics, Model: MK-VI) \\
\hline Soil organic carbon $(\%)$ & Walkley-Black chromic acid wet oxidation method \\
\hline
\end{tabular}


Table.2 Monthly variation (Mean $\pm \mathrm{SD}$ ) in water temperature $\left({ }^{\circ} \mathrm{C}\right)$ in different sites of Nalban wetland during the study period

\begin{tabular}{|l|l|l|l|l|l|l|}
\hline Site & SEPTEMBER & OCTOBER & NOVEMBER & DECEMBER & JANUARY & FEBRUARY \\
\hline Site 1 & $31.07 \pm 0.06^{\mathrm{el}}$ & $28.33 \pm 0.06^{\mathrm{cl}}$ & $29.10 \pm 0.10^{\mathrm{d} 2}$ & $21.13 \pm 0.15^{\mathrm{a} 1}$ & $21.07 \pm 0.12^{\mathrm{a} 1}$ & $23.00 \pm 0.10^{\mathrm{b} 1}$ \\
\hline Site 2 & $31.10 \pm 0.10^{\mathrm{bl}}$ & $28.47 \pm 0.06^{\mathrm{b} 2}$ & $28.70 \pm 0.10^{\mathrm{b} 1}$ & $21.07 \pm 0.12^{\mathrm{a} 1}$ & $21.07 \pm 0.12^{\mathrm{a} 1}$ & $23.17 \pm 0.15^{\mathrm{b} 1}$ \\
\hline Site 3 & $31.00 \pm 0.00^{\mathrm{d} 1}$ & $28.23 \pm 0.06^{\mathrm{cl}}$ & $28.83 \pm 0.06^{\mathrm{cl}}$ & $21.10 \pm 0.10^{\mathrm{a} 1}$ & $21.90 \pm 1.13^{\mathrm{a} 1}$ & $23.03 \pm 0.06^{\mathrm{b} 1}$ \\
\hline
\end{tabular}

Values with the same superscripts do not differ significantly $(\mathrm{P}>0.05)$.

Table.3 Monthly variation (Mean \pm SD) in water transparency $(\mathrm{cm})$ in different sites of Nalban wetland during the study period

\begin{tabular}{|c|c|c|c|c|c|c|}
\hline Site & SEPTEMBER & OCTOBER & NOVEMBER & DECEMBER & JANUARY & FEBRUARY \\
\hline Site 1 & $26.80 \pm 0.10^{\mathrm{b} 1}$ & $26.07 \pm 0.12^{\mathrm{c} 2}$ & $25.53 \pm 0.15^{\mathrm{dl}}$ & $22.50 \pm 0.10^{\mathrm{el}}$ & $20.07 \pm 0.12^{\mathrm{a} 1}$ & $19.97 \pm 0.15^{\mathrm{a} 2}$ \\
\hline Site 2 & $27.07 \pm 0.12^{\mathrm{a} 2}$ & $24.93 \pm 0.12^{\mathrm{b} 1}$ & $25.37 \pm 0.15^{\mathrm{c} 12}$ & $23.03 \pm 0.15^{\mathrm{d} 2}$ & $20.67 \pm 0.12^{\mathrm{e} 2}$ & $19.10 \pm 0.17^{\mathrm{fl}}$ \\
\hline Site 3 & $28.47 \pm 0.15^{\mathrm{e} 3}$ & $25.23 \pm 0.25^{\mathrm{bd} 1}$ & $25.17 \pm 0.15^{\mathrm{d} 1}$ & $23.23 \pm 0.21^{\mathrm{c} 2}$ & $21.13 \pm 0.15^{\mathrm{a} 3}$ & $22.07 \pm 0.12^{\mathrm{b} 3}$ \\
\hline
\end{tabular}

Values with the same superscripts do not differ significantly $(\mathrm{P}>0.05)$.

Table.4 Monthly variation (Mean \pm SD) in water $\mathrm{pH}$ in different sites of Nalban wetland during the study period

\begin{tabular}{|l|l|l|l|l|l|l|}
\hline \multicolumn{1}{|c|}{ Month } & SEPTEMBER & OCTOBER & NOVEMBER & DECEMBER & JANUARY & FEBRUARY \\
\cline { 1 - 5 } Site & & & & & \\
\hline Site 1 & $7.50 \pm 0.00^{\mathrm{c} 2}$ & $7.43 \pm 0.06 b^{\mathrm{cl}}$ & $7.40 \pm 0.00^{\mathrm{b} 1}$ & $7.27 \pm 0.06^{\mathrm{a} 1}$ & $7.63 \pm 0.06^{\mathrm{dl}}$ & $7.37 \pm 0.06^{\mathrm{b} 1}$ \\
\hline Site 2 & $7.60 \pm 0.00^{\mathrm{b} 1}$ & $7.57 \pm 0.06^{\mathrm{b} 2}$ & $7.50 \pm 0.00^{\mathrm{a} 2}$ & $7.47 \pm 0.06^{\mathrm{a} 2}$ & $7.80 \pm 0.00^{\mathrm{c} 2}$ & $7.60 \pm 0.00^{\mathrm{b} 3}$ \\
\hline Site 3 & $7.60 \pm 0.00^{\mathrm{bc1}}$ & $7.53 \pm 0.06^{\mathrm{ab} 12}$ & $7.57 \pm 0.06^{\mathrm{ab} 3}$ & $7.53 \pm 0.06^{\mathrm{ab} 2}$ & $7.67 \pm 0.06^{\mathrm{cl}}$ & $7.50 \pm 0.00^{\mathrm{a} 2}$ \\
\hline
\end{tabular}

Values with the same superscripts do not differ significantly $(\mathrm{P}>0.05)$. 
Table.5 Monthly variation (Mean \pm SD) in dissolved oxygen $(\mathrm{mg} / \mathrm{l})$ in different sites of Nalban wetland during the study period

\begin{tabular}{|l|l|l|l|l|l|l|}
\hline \multicolumn{1}{|c|}{ Month } & SEPTEMBER & OCTOBER & NOVEMBER & DECEMBER & JANUARY & FEBRUARY \\
\hline Site & & & & & \\
\hline Site 1 & $7.33 \pm 0.15^{\mathrm{al}}$ & $7.50 \pm 0.10^{\mathrm{a} 1}$ & $7.77 \pm 0.11^{\mathrm{b} 2}$ & $8.32 \pm 0.08^{\mathrm{cl}}$ & $8.52 \pm 0.10^{\mathrm{d} 2}$ & $8.17 \pm 0.05^{\mathrm{cl}}$ \\
\hline Site 2 & $7.43 \pm 0.15^{\mathrm{al}}$ & $7.36 \pm 0.06^{\mathrm{al}}$ & $7.61 \pm 0.08^{\mathrm{b} 2}$ & $8.23 \pm 0.10^{\mathrm{cl}}$ & $8.280 .04^{\mathrm{cl}}$ & $8.480 .11^{\mathrm{d} 2}$ \\
\hline Site 3 & $7.60 \pm 0.20^{\mathrm{bl}}$ & $7.37 \pm 0.12^{\mathrm{al}}$ & $7.28 \pm 0.04^{\mathrm{a} 1}$ & $8.27 \pm 0.11^{\mathrm{cl}}$ & $8.38 \pm 0.07^{\mathrm{cl}}$ & $8.25 \pm 0.07^{\mathrm{cl}}$ \\
\hline
\end{tabular}

Values with the same superscripts do not differ significantly $(\mathrm{P}>0.05)$.

Table.6 Monthly variation (Mean \pm SD) in free carbon dioxide $(\mathrm{mg} / \mathrm{l})$ in different sites of Nalban wetland during the study period

\begin{tabular}{|l|l|l|l|l|l|l|}
\hline \multicolumn{1}{|c|}{ Month } & SEPTEMBER & OCTOBER & NOVEMBER & DECEMBER & JANUARY & FEBRUARY \\
\hline Site & & & & & \\
\hline Site 1 & $16.30 \pm 0.10^{\mathrm{d} 1}$ & $12.63 \pm 0.06^{\mathrm{cl}}$ & $9.23 \pm 0.15^{\mathrm{b} 1}$ & $8.23 \pm 0.06^{\mathrm{a} 1}$ & $8.20 \pm 0.10^{\mathrm{a} 1}$ & $8.60 \pm 0.00^{\mathrm{el}}$ \\
\hline Site 3 & $17.20 \pm 0.10^{\mathrm{c} 2}$ & $13.27 \pm 0.06^{\mathrm{d} 2}$ & $10.20 \pm 0.35^{\mathrm{e} 2}$ & $8.70 \pm 0.10^{\mathrm{d} 2}$ & $8.53 \pm 0.12^{\mathrm{ab} 2}$ & $8.27 \pm 0.12^{\mathrm{a} 2}$ \\
\hline
\end{tabular}

Values with the same superscripts do not differ significantly $(\mathrm{P}>0.05)$.

Table.7 Monthly variation (Mean \pm SD) in total alkalinity $(\mathrm{mg} / \mathrm{l})$ in different sites of Nalban wetland during the study period

\begin{tabular}{|l|l|l|l|l|l|l|}
\hline Mite & SEPTEMBER & OCTOBER & NOVEMBER & DECEMBER & JANUARY & FEBRUARY \\
\hline Site 1 & $137.81 \pm 4.54^{\mathrm{a} 1}$ & $138.51 \pm 4.10^{\mathrm{a} 2}$ & $153.97 \pm 5.48^{\mathrm{b} 1}$ & $153.07 \pm 2.93^{\mathrm{b} 1}$ & $185.63 \pm 4.01^{\mathrm{c} 2}$ & $181.87 \pm 2.40^{\mathrm{c} 2}$ \\
\hline Site 2 & $138.30 \pm 3.80^{\mathrm{bl}}$ & $125.13 \pm 4.05^{\mathrm{cl}}$ & $150.83 \pm 4.66^{\mathrm{d} 1}$ & $163.60 \pm 4.90^{\mathrm{e} 2}$ & $186.30 \pm 2.88^{\mathrm{a} 2}$ & $185.70 \pm 2.25^{\mathrm{a} 2}$ \\
\hline Site 3 & $144.96 \pm 7.43^{\mathrm{bl}}$ & $129.63 \pm 4.10^{\mathrm{c} 1}$ & $111.07 \pm 2.27^{\mathrm{d} 2}$ & $160.00 \pm 3.96^{\mathrm{a} 12}$ & $166.03 \pm 3.21^{\mathrm{a} 1}$ & $162.70 \pm 1.30^{\mathrm{a} 1}$ \\
\hline
\end{tabular}

Values with the same superscripts do not differ significantly $(\mathrm{P}>0.05)$. 
Table.8 Monthly variation (Mean \pm SD) in total hardness $(\mathrm{mg} / \mathrm{l})$ in different sites of Nalban wetland during the study period

\begin{tabular}{|l|l|l|l|l|l|l|}
\hline \multicolumn{1}{|c|}{ Month } & SEPTEMBER & OCTOBER & NOVEMBER & DECEMBER & JANUARY & FEBRUARY \\
\hline Site 1 & $249.33 \pm 4.04^{\mathrm{a} 1}$ & $264.00 \pm 2.65^{\mathrm{bl}}$ & $271.00 \pm 2.65^{\mathrm{cl}}$ & $272.00 \pm 4.00^{\mathrm{c} 1}$ & $282.33 \pm 3.51^{\mathrm{d} 1}$ & $293.67 \pm 2.31^{\mathrm{el}}$ \\
\hline Site 2 & $256.00 \pm 2.00^{\mathrm{a} 2}$ & $268.67 \pm 1.15^{\mathrm{b} 2}$ & $274.00 \pm 3.00^{\mathrm{cl}}$ & $274.67 \pm 3.51^{\mathrm{c} 12}$ & $283.67 \pm 3.51^{\mathrm{d} 1}$ & $294.67 \pm 3.21^{\mathrm{el}}$ \\
\hline Site 3 & $260.67 \pm 1.15^{\mathrm{a} 2}$ & $274.00 \pm 2.00^{\mathrm{b3}}$ & $276.00 \pm 3.00^{\mathrm{bl}}$ & $281.00 \pm 2.65^{\mathrm{c} 2}$ & $294.00 \pm 2.00^{\mathrm{d} 2}$ & $296.33 \pm 4.04^{\mathrm{dl}}$ \\
\hline
\end{tabular}

Values with the same superscripts do not differ significantly $(\mathrm{P}>0.05)$

Table.9 Monthly variation (Mean \pm SD) in total dissolved solids (mg/l) in different sites of Nalban wetland during the studyperiod

\begin{tabular}{|l|l|l|l|l|l|l|}
\hline Site & SEPTEMBER & OCTOBER & NOVEMBER & DECEMBER & JANUARY & FEBRUARY \\
\hline Site 1 & $447.33 \pm 4.73^{\mathrm{a} 1}$ & $411.67 \pm 4.04^{\mathrm{b} 2}$ & $397.33 \pm 4.73^{\mathrm{c} 2}$ & $374.00 \pm 4.58^{\mathrm{d} 1}$ & $341.00 \pm 6.00^{\mathrm{e} 1}$ & $356.33 \pm 6.03^{\mathrm{f} 1}$ \\
\hline Site 2 & $463.67 \pm 6.66^{\mathrm{e} 2}$ & $418.00 \pm 5.29^{\mathrm{d} 2}$ & $408.00 \pm 6.56^{\mathrm{d} 2}$ & $386.00 \pm 6.00^{\mathrm{c} 2}$ & $356.33 \pm 6.51^{\mathrm{a} 2}$ & $368.33 \pm 3.79^{\mathrm{b} 2}$ \\
\hline Site 3 & $402.33 \pm 4.51^{\mathrm{d} 3}$ & $382.67 \pm 8.02^{\mathrm{cl}}$ & $366.00 \pm 8.72^{\mathrm{d} 1}$ & $354.33 \pm 3.06^{\mathrm{a} 3}$ & $367.00 \pm 7.55^{\mathrm{b} 2}$ & $386.33 \pm 5.13^{\mathrm{c} 3}$ \\
\hline
\end{tabular}

Values with the same superscripts do not differ significantly $(\mathrm{P}>0.05)$

Table.10 Monthly variation (Mean \pm SD) in electrical conductivity $(\mu \mathrm{s} / \mathrm{cm})$ in different sites of Nalban wetland during the study

\begin{tabular}{|l|l|l|l|l|l|l|}
\hline \multicolumn{7}{|c|}{ Meriod } \\
\hline Site & SEPTEMBER & OCTOBER & NOVEMBER & DECEMBER & JANUARY & FEBRUARY \\
\hline Site 1 & $1171.67 \pm 7.64^{\mathrm{a} 12}$ & $1155.00 \pm 5.00^{\mathrm{b} 1}$ & $1076.00 \pm 9.85^{\mathrm{cl}}$ & $1239.00 \pm 3.61^{\mathrm{d} 1}$ & $1221.33 \pm 4.16^{\mathrm{e} 1}$ & $1206.67 \pm 4.04^{\mathrm{fl}}$ \\
\hline Site 2 & $1182.00 \pm 4.00^{\mathrm{a} 2}$ & $1198.67 \pm 7.02^{\mathrm{b} 2}$ & $1123.33 \pm 6.11^{\mathrm{c} 2}$ & $1267.33 \pm 4.04^{\mathrm{b} 2}$ & $1257.33 \pm 6.03^{\mathrm{e} 2}$ & $1237.33 \pm 5.69^{\mathrm{f} 2}$ \\
\hline Site 3 & $1164.67 \pm 4.51^{\mathrm{al}}$ & $1201.67 \pm 5.13^{\mathrm{c} 2}$ & $1177.00 \pm 7.00^{\mathrm{d} 3}$ & $1211.67 \pm 3.79^{\mathrm{cd} 3}$ & $1244.33 \pm 7.64^{\mathrm{e} 3}$ & $1218.67 \pm 7.02^{\mathrm{d} 3}$ \\
\hline
\end{tabular}

Values with the same superscripts do not differ significantly $(\mathrm{P}>0.05)$ 
Table.11 Monthly variation (Mean \pm SD) in Biological Oxygen Demand (mg/l) in different sites of Nalban wetland during the study period

\begin{tabular}{|l|l|l|l|l|l|l|}
\hline \multicolumn{1}{|c|}{ Month } & SEPTEMBER & OCTOBER & NOVEMBER & DECEMBER & JANUARY & FEBRUARY \\
\hline Site & & & & & \\
\hline Site 1 & $16.87 \pm 1.67^{\mathrm{a} 1}$ & $18.47 \pm 1.02^{\mathrm{a} 1}$ & $21.83 \pm 1.76^{\mathrm{b} 1}$ & $19.57 \pm 1.01^{\mathrm{ab} 1}$ & $17.00 \pm 1.61^{\mathrm{a} 1}$ & $18.50 \pm 1.50^{\mathrm{a} 1}$ \\
\hline Site 2 & $21.77 \pm 1.32^{\mathrm{b} 2}$ & $17.50 \pm 0.89^{\mathrm{a} 1}$ & $25.27 \pm 0.95^{\mathrm{c} 2}$ & $23.03 \pm 0.96^{\mathrm{b} 2}$ & $27.23 \pm 0.87^{\mathrm{d} 2}$ & $29.17 \pm 1.04^{\mathrm{e} 2}$ \\
\hline Site 3 & $28.53 \pm 0.75^{\mathrm{a} 3}$ & $28.50 \pm 1.5^{\mathrm{a} 2}$ & $31.50 \pm 0.50^{\mathrm{b} 3}$ & $28.57 \pm 0.40^{\mathrm{a} 3}$ & $33.17 \pm 1.04^{\mathrm{c} 3}$ & $30.23 \pm 0.87^{\mathrm{ab} 2}$ \\
\hline
\end{tabular}

Values with the same superscripts do not differ significantly $(\mathrm{P}>0.05)$.

Table.12 Monthly variation (Mean $\pm \mathrm{SD}$ ) in nitrate-nitrogen $(\mathrm{mg} / \mathrm{l})$ in different sites of Nalban wetland during the study period

\begin{tabular}{|l|l|l|l|l|l|l|}
\hline \multicolumn{1}{|c|}{ Month } & SEPTEMBER & OCTOBER & NOVEMBER & DECEMBER & JANUARY & FEBRUARY \\
\cline { 1 - 6 } Site & $0.083 \pm 0.012^{\mathrm{bcl}}$ & $0.092 \pm 0.005^{\mathrm{cl}}$ & $0.070 \pm 0.003^{\mathrm{ab} 1}$ & $0.091 \pm 0.007^{\mathrm{cl}}$ & $0.065 \pm 0.008^{\mathrm{a} 1}$ & $0.083 \pm 0.010^{\mathrm{bc} 2}$ \\
\hline Site 1 & $0.083 \pm 0.005^{\mathrm{cd} 1}$ & $0.073 \pm 0.004 \mathrm{~b}^{\mathrm{c} 2}$ & $0.069 \pm 0.008^{\mathrm{dl}}$ & $0.085 \pm 0.005^{\mathrm{d} 1}$ & $0.053 \pm 0.008^{\mathrm{a} 1}$ & $0.067 \pm 0.005^{\mathrm{b} 1}$ \\
\hline Site 2 & $0.114 \pm 0.014^{\mathrm{a} 2}$ & $0.082 \pm 0.004^{\mathrm{a} 3}$ & $0.078 \pm 0.003^{\mathrm{a} 1}$ & $0.145 \pm 0.041^{\mathrm{b} 2}$ & $0.083 \pm 0.005^{\mathrm{a} 2}$ & $0.087 \pm 0.006^{\mathrm{a} 2}$ \\
\hline
\end{tabular}

Values with the same superscripts do not differ significantly $(\mathrm{P}>0.05)$.

Table.13 Monthly variation (Mean \pm SD) in ammonia-nitrogen $(\mathrm{mg} / \mathrm{l})$ in different sites of Nalban wetland during the study period

\begin{tabular}{|l|l|l|l|l|l|l|}
\hline \multicolumn{1}{|c|}{ Month } & SEPTEMBER & OCTOBER & NOVEMBER & DECEMBER & JANUARY & FEBRUARY \\
\hline Site & & & & & \\
\hline Site 1 & $0.29 \pm 0.02^{\mathrm{a} 1}$ & $0.41 \pm 0.02^{\mathrm{b} 1}$ & $0.36 \pm 0.03^{\mathrm{b} 1}$ & $0.39 \pm 0.03^{\mathrm{b} 1}$ & $0.48 \pm 0.03^{\mathrm{c} 2}$ & $0.52 \pm 0.06^{\mathrm{c} 2}$ \\
\hline Site 2 & $0.39 \pm 0.03^{\mathrm{ab} 2}$ & $0.37 \pm 0.08^{\mathrm{a} 1}$ & $0.45 \pm 0.04^{\mathrm{bcd} 2}$ & $0.41 \pm 0.03^{\mathrm{abc} 1}$ & $0.48 \pm 0.02^{\mathrm{cd} 2}$ & $0.51 \pm 0.03^{\mathrm{d} 12}$ \\
\hline Site 3 & $0.29 \pm 0.03^{\mathrm{a} 1}$ & $0.35 \pm 0.04^{\mathrm{b} 1}$ & $0.32 \pm 0.03^{\mathrm{ab} 1}$ & $0.41 \pm 0.02^{\mathrm{cl}}$ & $0.31 \pm 0.02^{\mathrm{ab} 1}$ & $0.43 \pm 0.03^{\mathrm{cl}}$ \\
\hline
\end{tabular}

Values with the same superscripts do not differ significantly $(\mathrm{P}>0.05)$. 
Table.14 Monthly variation (Mean \pm SD) in phosphate-phosphorous (mg/l) in different sites of Nalban wetland during the study period

\begin{tabular}{|l|l|l|l|l|l|l|}
\hline \multicolumn{1}{|c|}{ Month } & SEPTEMBER & OCTOBER & NOVEMBER & DECEMBER & JANUARY & FEBRUARY \\
\cline { 1 - 6 } Site & $0.08 \pm 0.00^{\mathrm{al}}$ & $0.15 \pm 0.02^{\mathrm{cl}}$ & $0.32 \pm 0.01^{\mathrm{el}}$ & $0.11 \pm 0.01^{\mathrm{b} 1}$ & $0.21 \pm 0.02^{\mathrm{d} 1}$ & $0.08 \pm 0.01^{\mathrm{a} 1}$ \\
\hline Site 1 & $0.11 \pm 0.01^{\mathrm{a} 1}$ & $0.25 \pm 0.01^{\mathrm{b} 2}$ & $0.36 \pm 0.01^{\mathrm{c} 2}$ & $0.17 \pm 0.01^{\mathrm{d} 2}$ & $0.31 \pm 0.02^{\mathrm{e} 2}$ & $0.14 \pm 0.01^{\mathrm{fl}}$ \\
\hline Site 2 & $0.09 \pm 0.00^{\mathrm{a} 2}$ & $0.20 \pm 0.01^{\mathrm{a} 3}$ & $0.30 \pm 0.02^{\mathrm{a} 1}$ & $0.13 \pm 0.02^{\mathrm{a} 3}$ & $0.21 \pm 0.02^{\mathrm{a} 1}$ & $0.102^{2} \pm 0.50^{\mathrm{al}}$ \\
\hline
\end{tabular}

Values with the same superscripts do not differ significantly $(\mathrm{P}>0.05)$.

Table.15 Monthly variation (Mean \pm SD) in soil $\mathrm{pH}$ in different sites of Nalban wetland during the study period

\begin{tabular}{|c|c|c|c|c|c|c|}
\hline Site & SEPTEMBER & OCTOBER & NOVEMBER & DECEMBER & JANUARY & FEBRUARY \\
\hline Site 1 & $7.70 \pm 0.10^{b 1}$ & $8.17 \pm 0.12^{\mathrm{dl}}$ & $7.90 \pm 0.10^{\mathrm{cl}}$ & $7.73 \pm 0.12^{\mathrm{bcl}}$ & $7.30 \pm 0.10^{\mathrm{a} 1}$ & $7.60 \pm 0.10^{\mathrm{b} 1}$ \\
\hline Site 2 & $7.63 \pm 0.15^{\mathrm{abl}}$ & $8.07 \pm 0.15^{\mathrm{dl}}$ & $7.90 \pm 0.10^{\mathrm{cd} 1}$ & $7.77 \pm 0.15^{\mathrm{bc} 12}$ & $7.40 \pm 0.10^{\mathrm{a} 1}$ & $7.60 \pm 0.10^{\mathrm{abl}}$ \\
\hline Site 3 & $7.80 \pm 0.10^{\mathrm{abl}}$ & $8.17 \pm 0.12^{\mathrm{cl}}$ & $7.90 \pm 0.10^{\mathrm{abl}}$ & $8.00 \pm 0.10^{b c 2}$ & $7.70 \pm 0.10^{\mathrm{a} 2}$ & $7.83 \pm 0.12^{\mathrm{ab} 2}$ \\
\hline
\end{tabular}

Values with the same superscripts do not differ significantly $(\mathrm{P}>0.05)$

Table.16 Monthly variation (Mean \pm SD) in organic carbon in different sites of Nalban wetland during the study period

\begin{tabular}{|l|l|l|l|l|l|l|}
\hline \multicolumn{1}{|c|}{ Month } & SEPTEMBER & OCTOBER & NOVEMBER & DECEMBER & JANUARY & FEBRUARY \\
\hline Site & $1.88 \pm 0.09^{\mathrm{a} 2}$ & $2.36 \pm 0.09^{\mathrm{c} 2}$ & $1.78 \pm 0.09^{\mathrm{a} 1}$ & $1.89 \pm 0.05^{\mathrm{a} 1}$ & $2.43 \pm 0.07^{\mathrm{c} 1}$ & $2.10 \pm 0.12^{\mathrm{b} 1}$ \\
\hline Site 1 & $1.65 \pm 0.12^{\mathrm{a} 1}$ & $1.97 \pm 0.17^{\mathrm{bl}}$ & $1.78 \pm 0.10^{\mathrm{ab} 1}$ & $1.67 \pm 0.09^{\mathrm{a} 2}$ & $2.28 \pm 0.15^{\mathrm{cl}}$ & $1.97 \pm 0.04^{\mathrm{d} 1}$ \\
\hline Site 2 & $1.83 \pm 0.04^{\mathrm{a} 2}$ & $2.46 \pm 0.09^{\mathrm{c} 2}$ & $1.85 \pm 0.06^{\mathrm{al}}$ & $2.12 \pm 0.13^{\mathrm{d} 3}$ & $2.48 \pm 0.09^{\mathrm{cl}}$ & $2.35 \pm 0.06^{\mathrm{c} 2}$ \\
\hline
\end{tabular}

Values with the same superscripts do not differ significantly $(\mathrm{P}>0.05)$ 
Map.1 \& 2 Map of West Bengal \& Map of East Kolkata Wetland
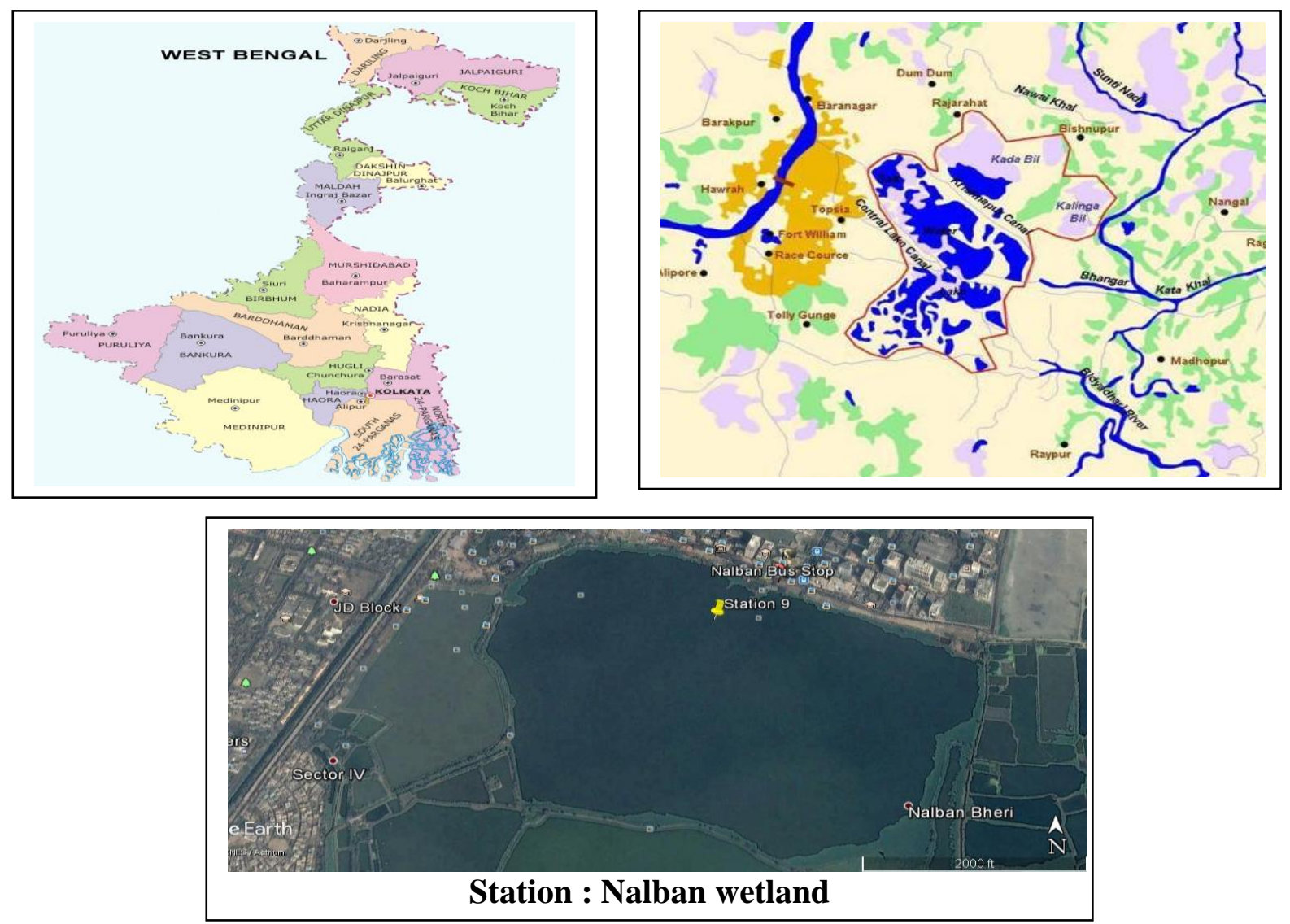

\section{Total organic carbon}

There was significant $(\mathrm{p}<0.05)$ difference between all three sites. September, November and December showed no significant $(\mathrm{p}>0.05)$ difference in between them but October, January and February months showed significant $(\mathrm{p}<0.05)$ difference with above mentioned months (Table 16). Siddique et al., (2012) stated that 6-8.4\% of organic matter were found from (1-5) years aged ponds and $10.6-11.3 \%$ and $12.9-13.4 \%$ organic matter were found from (6-10) years and above 10 years aged ponds respectively.

In conclusion, the present study suggests that the important physico-chemical parameters of water such as temperature, $\mathrm{pH}$, dissolved oxygen, free carbon dioxide, total alkalinity, total dissolved solids, electrical conductivity,
Biological oxygen demand, nitrate-nitrogen, ammonia-nitrogen, phosphate-phosphorous and soil quality parameters such as $\mathrm{pH}$ and organic carbon were studied in Nalban wetland and they were found to be within the tolerable limits of fishes that inhabit in the wetland. In view of the growing threats like uncontrolled siltation, unregulated discharge of waste water, industrial effluents, surface run-off and encroachment a holistic approach is the need of the hour for sustainable utilisation of this unique ecosystem.

\section{Acknowledgement}

The authors thankfully acknowledged the support and facilities provided by Dean, Faculty of Fishery Sciences, WBUAFS, Kolkata-97. 


\section{References}

Adams, G. (1988). Wetlands of the prairies of Canada. Wetlands of Canada, pp. 155198.

Al-jaboobi, M., El-ariqi, S., El Housni, A., Zouahri, A. and Bouksaim, M. (2013). Assessment of the impact of wastewater use on soil properties. Journal of Materials and Environmental Science, 5(3): 747-752.

Ayyappan, S., Jena, J.K., Gopalakrishnan, A. and Pandey, A.K. (2006). Hand book of fisheries and aquaculture. published by Directorate of Knowledge Management in Agriculture, ICAR. pp. 591-622.

Basu, A., Sengupta, S., Dutta, S., Saha, A., Ghosh, P. and Roy, S., 2013. Studies on macrobenthic organisms in relation to water parameters at East Calcutta Wetlands. Journal of Environmental Biology, 34(7): 733-737.

Bhatnagar, A., Jana, S.N., Garg, S.K. Patra, B.C., Singh, G. and Barman, U.K. (2004).Water quality management in aquaculture, In: Course Manual of summer school on development of sustainable aquaculture technology in fresh and saline waters, CCS Haryana Agricultural, Hisar (India), pp 203- 210.

Bhowmik, M. L., Jena, J.K. and Ayyappan, S. (2011). Waste water aquaculture. In: Hand book of fisheries and aquaculture, published by Directorate of knowledge management in agriculture, ICAR, New Delhi, pp.449-468.

Boyd, C.E. and Lichtkoppler, F. (1979). Water Quality Management in Fish Ponds. Research and Development series, 22: 45-47.

Chase, J.M. (2007). Drought mediates the importance of stochastic community assembly. Proceedings of the National Academy of Sciences, 104(44): 1743017434.

Chatterjee, N.R., Sahoo, D. and Chetri, C.
(2014). Study on the monthly variation in hydro biological condition and its relation to fish production of a sewage fed Bheri system at suburban Kolkata. Journal of Aquaculture Research and Development, 5(7:100279): 1-8.

Chattopadhyay, G. N., Saha, P. K., Ghosh, A., and Karmakar, H. C. (1988). A study on optimum BOD levels for fish culture in wastewater ponds. Biological Wastes, 25: 79- 83.

Dampin, N., Tarnchalanukit, W., Chunkao, K. and Maleewong, M. (2012). Fish Growth Model for Nile Tilapia (Oreochromis niloticus) in Wastewater Oxidation Pond, Thailand. Procedia Environmental Sciences, 8: 513-524.

Dasgupta, S., Pandey, B.K., Sarangi, N. and Mukhopadhyay P.K. (2008). Evaluation of water productivity and fish yield in sewage-fed vis-a`-vis fertilized based carp culture. Bioresource Technology, 99: 3499-3505.

Ghosh, A., Maity, B., Chakrabarti, K. and Chattopadhyay, D. (2007). Bacterial diversity of east Calcutta wet land area: possible identification of potential bacterial population for different biotechnological uses. Microbial Ecology, 54(3): 452-459.

Jena, J. and Das, P.C. (2011). Grow-out performance of Kuria labeo, Labeo gonius (Hamilton), with major carps in carp polyculture system. Aquaculture Research, 42(9): 1332-1338.

Mandal, B., Some, S. and Banerjee, B. (2010). Comparative studies on primary productivity of sewage fed freshwater fishpond in relation to production efficiency of phytoplankton. International Journal of science and nature, 1(1): 89-94.

Meade, J.W. (1985). Allowable ammonia for fish culture. Progressive Fish culture, 47: 135- 145.

Mould, D.J., Frahm, E., Salzmann, T., 
Miegel, K. and Acreman, M.C. (2010). Evaluating the use of diurnal groundwater fluctuations for estimating evapotranspiration in wetland environments: case studies in southeast England and northeast Germany. Ecohydrology, 3(3): 294-305.

Mukherjee, D. P. (2011). Stress of urban pollution on largest natural wetland ecosystem in East Kolkata-causes, consequences and improvement. Scholars research library, Archives of Applied Science Research, 3(6): 443-461.

Nwabueze, A. A. (2013). Growth Performance of the Mudfish, Clarias anguillaris (Pellegrin, 1923) in Treated and Untreated Domestic Sewage, Sustainable Agriculture Research. Canadian Center of Science and Education, 2(1): 62-69.

Panthi, S., Dhakal, M., Thagunna, S.S. and Thapa, B.B. (2014). Status of Wetland in Dhorpatan Hunting Reserve, Nepal. Open Journal of Ecology, 4(05): 245.

Saha, K.C. and Majumdar, B. (1970). Fisheries of West Bengal. Superintendent, Government, Print., West Bengal Government Press. 35(5): 216-217.

Santhosh, B. and Singh, N. P. (2007). Guidelines for water quality management for fish culture in Tripura, ICAR Research Complex for NEH Region,
Tripura Center, Publication no.29.

Santra, S.C. and Deb, S.C. (1996). Hydrochemistry and hydrobiology of sewage fed jheels in the tropics: a case study. Indian Hydrobiology, 1: 5-17.

Siddique, M.A.M., Barua, P. and Ghani, M.H. (2012). Comparative study of physicochemical properties of soil according to the age of aquaculture pond of Bangladesh. Mesopotamian Journal of Marine Science, 27(1): 29-38.

Sondhia, S. (2008). Evaluation of SewageFed Fisheries in Terms of water quality Risk of Heavy metal contamination and Fish Yield., Proceedings of Taal 2007: The 12thWorld Lake Conference edited by Sengupta, M. and Dalwani, R., pp.165-166.

Thang, V.Q. and Yen, M. D. (2003). Wastewater-fed aquaculture in the wetlands of Hanoi, status and problems. Osaka University Knowledge Archieve, Bulletin of Universities, Ministry of Training and Education, pp. 60-66.

Williams, M. (1990). Understanding Wetlands In: Wetlands: A threatened Landscape. Michael Williams, Basil Blakewell Ltd., Oxford, pp. 1-3.

Zar, J. H. (1999). Biostatistical analysis. Pearson Education India, 4th edition. pp. 3-10.

\section{How to cite this article:}

Ranga Ram Mohan, T.S. Nagesh, Anish Das, Sandeep Sahu, D. Ravindra Kumar Reddy and Anand Prasad Paturi. 2019. Physico-Chemical Characteristics of Nalban Wetland of East Kolkata Wetlands, A Ramsar Site, West Bengal, India. Int.J.Curr.Microbiol.App.Sci. 8(04): 1264-1275. doi: https://doi.org/10.20546/ijcmas.2019.804.145 